\title{
Anomalías en el desarrollo del cuarto aparato faríngeo como causa de infecciones profundas del cuello recidivantes
}

\author{
Anomalies in the fourth pharyngeal apparatus as cause \\ of deep recidivant neck infections
}

\author{
María de los Ángeles Contreras $\mathrm{R}^{1}$, José Miguel Contreras $\mathrm{R}^{2}$, \\ Eduardo Reyes $\mathrm{S}^{1}$, Alejandro Paredes $\mathrm{W}^{2}$.
}

\begin{abstract}
RESUMEN
La mayoría de las infecciones profundas del cuello se originan a partir de un foco en la mucosa aerodigestiva superior o a partir de un foco dental, las anomalías congénitas corresponden sólo a $2 \%$ de las causas.

La presentación clínica es bastante similar independientemente de la etiología y en muchas ocasiones no basta con la clínica para llegar a un correcto diagnóstico etiológico.

La presencia de una infección del espacio profundo del cuello (IEPC) a repetición es altamente sugerente de una anomalía congénita de las cuales aproximadamente el 58\% derivan del aparato faríngeo. De éstas, las provenientes del IV aparato faríngeo son extremadamente raras siendo más frecuentes en la niñez, en el lado izquierdo del cuello y en pacientes de sexo femenino.

Estas IEPC de causa congénita también tienen una buena respuesta a la terapia antibiótica y/o al drenaje por lo tanto ello lo enmascara como un factor etiológico.

Realizar el diagnóstico de anomalía del desarrollo del IV aparato faríngeo es difícil. Elas son poco frecuente (1-2\% de las anomalías congénitas) y clínicamente se presentan como abscesos recurrentes del espacio profundo del cuello, al lado izquierdo, generalmente en la niñez, acompañados o no de una tiroiditis aguda supurada izquierda asociado al hallazgo del orificio persistente (seno) en el ápex del seno piriforme.

Se comunican 3 pacientes con absceso cervical izquierdo secundario a una anomalía en el desarrollo del IV aparato faríngeo.
\end{abstract}

Palabras clave: Absceso cervical, anomalía congénita, seno IV bolsa faríngea.

\section{SUMMARY}

Most of the deep neck infections originate from a focus in the upper aerodigestive mucous or from a dental focus, congenital anomalies only correspond to a $2 \%$ of the causes.

\footnotetext{
1 Internos Escuela de Medicina, Universidad de Chile.

${ }^{2}$ Médico del Servicio de Otorrinolaringología, Hospital San Juan de Dios, Universidad de Chile.
} 
Cinical presentation is quite similar independently from the etiology and in many occasions clinic is not sufficient to reach a correct etiological diagnosis.

The presence of a repeated infection of the deep space in the neck (IEPC) is highly suggestive of a congenital anomaly of which approximately 58\% derive from the pharyngeal apparatus. From these, those coming from the IV pharyngeal apparatus are extremely rare, being more frequent in childhood, in the left side of the neck and in female patients.

These congenital IEPC also show good response to antibiotic therapy and/or drainage that is why it masks it as an etiological factor.

It is difficult to make a diagnosis of pharyngeal apparatus IV development abnormality. They are infrequent (1-2\% of the congenital abnormalities) and are clinically presented as recurrent abscesses of the deep space of the neck, to the left hand side, generally during childhood, accompanied or not by an acute left side suppurating thyroiditis associated to a persisting finding of the cavity in the apex of the pyriform cavity.

3 patients are reported with left side cervical abscess secondary to an anomaly in the development of the pharyngeal apparatus IV.

Key words: Cervical abscess, congenital anomaly, IV cavity of the pharyngeal bourse.

\section{INTRODUCCIÓN}

La mayoría de las infecciones del espacio profundo del cuello (IEPC) son el resultado de una adenitis supurativa. La localización del foco primario es comúnmente la vía aerodigestiva superior o un foco dental. En forma menos común se deben a perforación por cuerpo extraño, tromboflebitis de la vena yugular interna, osteomielitis o anomalías congénitas.

Las I I C cuya causa sean las anomalías congénitas del desarrollo de la cabeza y el cuello sólo corresponden a $2 \%{ }^{1}$ y clínicamente deben sospecharse frente a recurrencias del cuadro infeccioso.

De todas las anomalías congénitas responsables de las IEPC recurrentes, aproximadamente $58 \%$ se deben a una alteración en el desarrollo del aparato faríngeo ${ }^{2}$. Noventa y cinco por ciento de las anomalías del aparato faríngeo (branquial) se originarían del II aparato; del $4 \%-5 \%$ del I y III. Las provenientes del IV aparato faríngeo son extremadamente raras (1\%-2\%) 3 .

Para realizar un diagnóstico de anomalía del IV aparato faríngeo se requiere de un alto índice de sospecha asociado a un estudio imagenológico adecuado y de un examen endoscópico bajo anestesia general, sólo de esta forma se confirmará la presencia del orificio remanente del conducto faringo-branquial en el ápex del seno piriforme para así realizar el tratamiento adecuado a esta entidad evitando la recurrencia del cuadro.

Se comunican 3 casos clínicos tratados en el Servicio de Otorrinolaringología del Hospital San Juan de Dios, de sexo femenino que presentan IEPC a izquierda cuya etiología correspondían a anomalías en el desarrollo del IV aparato faríngeo.

\section{CASOS CLÍNICOS}

\section{Caso № 1}

Paciente sexo femenino de 10 años de edad con antecedentes de hipotiroidismo y de dos cuadros de tiroiditis aguda supurativa izquierda, manejada con antibioticoterapia y drenaje quirúrgico. Es evaluada por Otorrinolaringología el 17/07/2000 en su tercer cuadro de IEPC a izquierda. Posee estudio 
ecográfico que evidencia un aumento global de la glándula tiroides heterogénea asociado a borramiento del plano del músculo ECM ipsilateral y rotura de la cápsula de la glándula que comunica a un área hipoecogénica paralaringo-traqueal. Se sospecha anomalía congénita proveniente del tercer o cuarto aparato faríngeo y se realiza un tránsito esofágico que fue negativo en la búsqueda de remanente del tracto faringo-branquial. Una microlaringoscopía directa evidencia la existencia de un orificio en el ápex del seno piriforme izquierdo. En julio/2000 se realiza una cervicotomía exploradora resecándose el tejido fibroso entre tiroides y el área retrolaríngea e hipofaringe. En este caso no se evidenció tracto sinusal.

Evoluciona en forma satisfactoria, sin complicación y es dada de alta con diagnóstico de seno de la IV bolsa faríngea. $\boxminus$ seguimiento a seis años no evidencia recidiva y actualmente se encuentra en tratamiento por el hipotiroidismo secundario a la infección.

\section{Caso № 2}

Paciente sexo femenino de 4 años de edad $\sin$ antecedentes mórbidos que consulta por una IEPC a izquierda que surge después de un cuadro infeccioso de vía respiratoria alta (septiembre/2004). Posee TC que evidencia una masa de $3 \mathrm{~cm}$ en el lóbulo tiroideo izquierdo y espacio circundante que desplaza la vía aérea. Se produce el drenaje espontáneo por boca. En la endoscopía flexible nasofa-

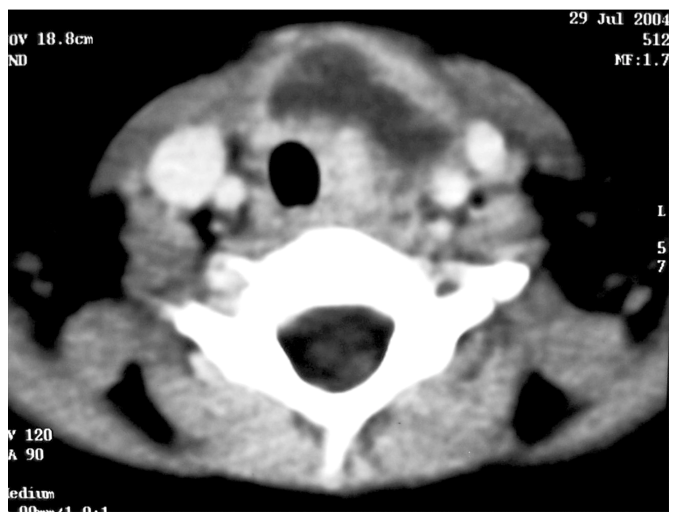

Figura 1. Caso №3. TC de cuello con medio de contraste: imagen hipodensa anterolateral a glándula tiroidea izquierda. ringolaríngea (NR) no se evidenció orificio sinusal. $\boxminus$ tránsito esofágico revela un trayecto ciego que se origina en ápex de seno piriforme izquierdo. Se maneja cuadro agudo con antibioticoterapia y se planifica su cirugía dos meses después del cuadro agudo con diagnóstico de persistencia del tracto faringo-branquial del IV aparato faríngeo. TC de control preoperatorio sin evidencia de inflamación. La cirugía se realiza en noviembre/2004; previa microlaringoscopía directa en la cual se evidencia el trayecto "fistuloso" en el ápex del seno piriforme izquierdo. Mediante cervicotomía izquierda se reseca el trayecto fistuloso y lóbulo tiroideo izquierdo desinsertando el cuerno inferior del cartílago tiroides para exponer el seno piriforme. Evoluciona con paresia cordal izquierda transitoria y su seguimiento a 3 meses, sin recidiva y a dos años sin reingreso.

\section{Caso $N^{\circ} 3$}

Paciente 26 años, sexo femenino, sin antecedentes mórbidos conocidos quien tiene historia de IEPC izquierdo a repetición (en 4 oportunidades) desde noviembre/2005, cuadros que fueron manejados con AINES, antimicrobianos y drenaje quirúrgico (en último cuadro) con el diagnóstico presuntivo de tiroiditis supurativa izquierda. $日$ cultivo es (+) a flora respiratoria y el TC de cuello revela un aumento de volumen hipoecogénico extenso cervical izquierdo, concordante con absceso que comprime la tráquea (Fgura 1).

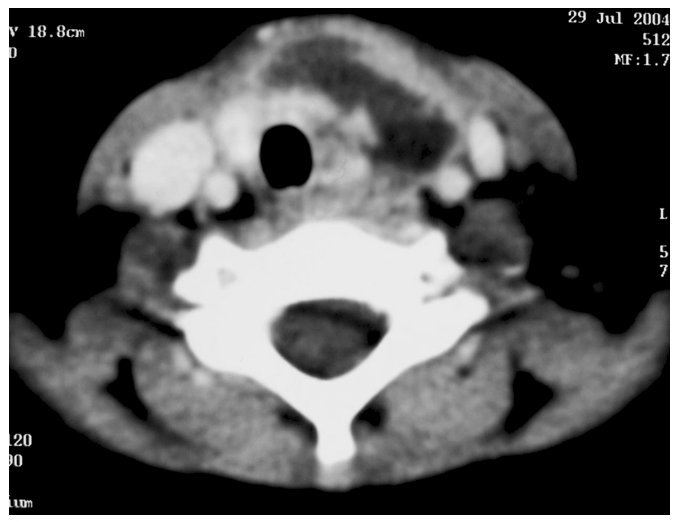

Caso №3. TC de cuello con medio de contraste: imagen hipodensa anterolateral a glándula tiroidea izquierda. 
Derivada a Otorrinolaringología se evalúa con $\mathrm{NF}$ que es dudosa para la presencia de orificio sinusal en seno piriforme izquierdo. Se solicita tránsito esofágico que demuestra la presencia de un trayecto ciego en el ápex del seno piriforme izquierdo. Se diagnostica seno de la IV bolsa faríngea izquierdo planificándose su manejo mediante cauterización endoscópica del seno persistente.

\section{DISCUSIÓN}

Las anomalías en el desarrollo del aparato faríngeo (término más adecuado que branquial) debido a la falla en la fusión de sus componentes a la $7^{a}$ semana (bolsa, hendidura y arco) se pueden clasificar en: quistes, senos y fístulas (Figuras 2, 3 y 4).

Los quistes son cavidades que no se comunican con el exterior (ya sea a faringe o piel) y pueden originarse de la bolsa faríngea, hendidura faríngea o del seno cervical. Las fístulas corres-

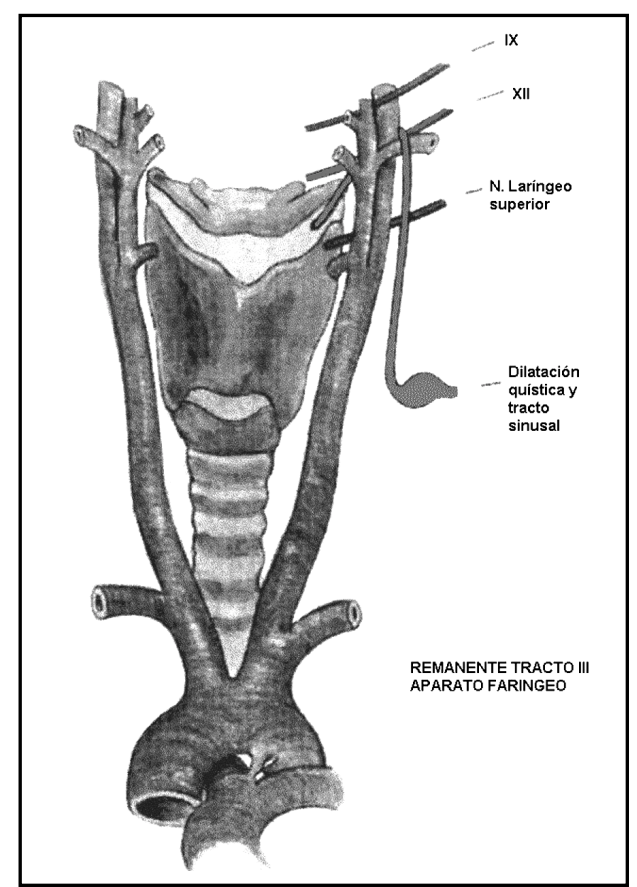

Figura 3. III Aparato faríngeo.

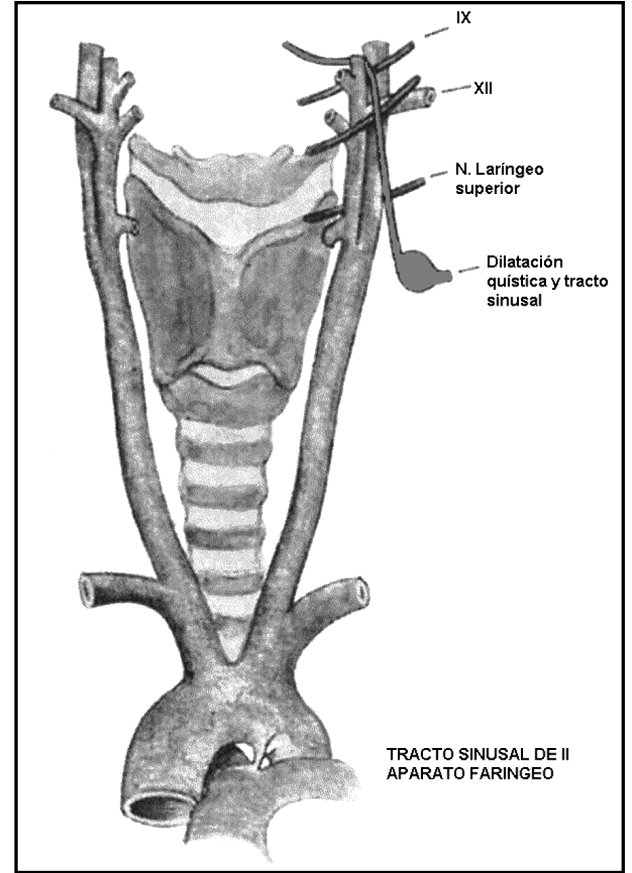

Figura 2. II Aparato faríngeo.

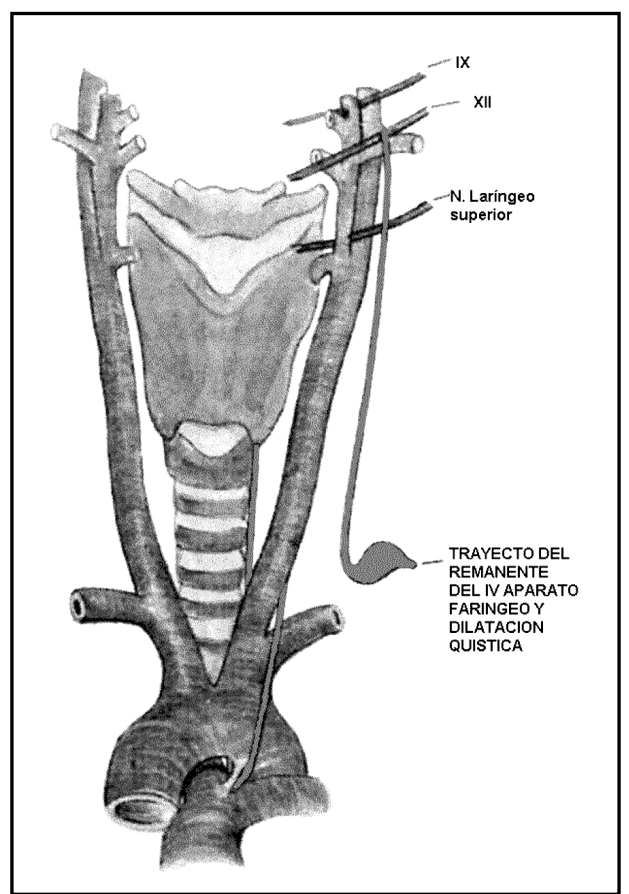

Figura 4. IV Aparato faríngeo. 
ponden a trayectos que comunican la faringe (orificio interno) con la piel (orificio externo), se originan por la persistencia de la bolsa y la hendidura faríngea y pueden o no estar asociado a un quiste. 日 seno tiene un extremo ciego que puede tener su orificio en faringe (persistencia de una bolsa faríngea) 0 en piel (persistencia de la hendidura faríngea).

Las anomalías del IV aparato faríngeo son bastante raras y corresponden a tan sólo el $1 \%$ a $2 \%{ }^{3-5}$, ellas pueden clasificarse en:

a) Quiste: son poco frecuentes y se presentan como un nódulo tiroideo "frío" o absceso ${ }^{6}$.

b) Fistula: la presencia de una fístula del IV aparato faríngeo a derecha nunca ha sido reportada en la literatura ${ }^{5-8}$. La ruta posible que debiera tener una fístula del IV aparato faríngeo comenzaría en el ápex del seno piriforme y se extendería inferiormente hasta salir de la faringe caudal al nervio laríngeo superior, músculo cricotiroideo y cartílago tiroideo. Luego el trayecto continuaría su curso lateral a la tráquea y el nervio laríngeo recurrente. En las fístulas del lado izquierdo el tracto baja hasta el arco aórtico y en esta localización realiza un asa ascendiendo por el cuello, posterior a la arteria carótida común. De allí la fístula realiza otra asa alrededor del nervio hipogloso y desciende nuevamente hasta llegar al orificio de salida en la piel (anterior al tercio inferior del músculo esternocleidomastoideo). A derecha el asa es alrededor del tronco braquiocefálico $3,6-7$.

c) Seno: diferenciar un seno proveniente del III ó IV aparato faríngeo es difícil puesto que ambos desembocan en el seno piriformey presentan un recorrido similar antes de terminar en la región paratraqueal o glándula tiroidea 4 . Eementos que pueden ayudar en el diagnóstico diferencial son la relación del trayecto del seno con el nervio laríngeo superior, en caso de origen en la IV bolsa faríngea el trayecto ciego cursa caudal al nervio laríngeo superior y cefálico al nervio laríngeo recurrente, mientras que en aquellos casos derivados del III aparato faríngeo el tra yecto se ubica sobre el nervio laríngeo superior. Aro elemento es la ubicación del orificio ciego en el seno piriforme, ubicándose en el ápex en aquellos casos originados de la IV bolsa faríngea y en la base del seno piriforme en los casos originados en la III bolsa faríngea. La histología no es concluyente para realizar el diagnóstico diferencial entre seno de III ó IV bolsa faríngea. La presencia de tejido tímico ectópico y de glándula paratiroides era sugerente de anomalía de III bolsa, sin embargo no es categórico puesto que se han reportado casos de tejido tímico accesorio y la glándula paratiroides superior se de la IV bolsa faríngea4 ${ }^{4,9}$. (Tabla 1).

Tabla 1. Diferencias entre seno de la III y IV bolsa faríngea

\begin{tabular}{|lll|}
\hline Característica & Seno III bolsa faríngeo & Seno IV bolsa faríngea \\
\hline $\begin{array}{l}\text { Relación del tracto } \\
\text { anómalo con nervio } \\
\text { laríngeo superior y } \\
\text { laríngeo recurrente }\end{array}$ & $\begin{array}{l}\text { Oursa cefálico al nervio } \\
\text { laríngeo superior y } \\
\text { músculo constrictor } \\
\text { inferior }\end{array}$ & $\begin{array}{l}\text { arsa caudal al nervio } \\
\text { laríngeo superior y } \\
\text { cefálico al nervio } \\
\text { laríngeo recurrente }\end{array}$ \\
$\begin{array}{l}\text { Origen en relación a } \\
\text { seno piriforme }\end{array}$ & $\begin{array}{l}\text { Base o pared lateral } \\
\text { del seno piriforme }\end{array}$ & Apex seno piriforme \\
Histopatología & $\begin{array}{l}\text { Presencia de tejido } \\
\text { tímico ectópico y tejido } \\
\text { paratiroideo }\end{array}$ & $\begin{array}{l}\text { Presencia de tejido } \\
\text { tiroídeo, tejido tímico } \\
\text { accesorio y paratiroides } \\
\text { superior }\end{array}$ \\
\hline
\end{tabular}


Cínicamente el seno del IV aparato faríngeo se caracteriza por ser diagnosticada principalmente en la niñez, ser más frecuente en el sexo femenino y presentarse en el $97 \%$ de los casos en el lado izquierdo $5,6,9$. En neonatos se presenta como masa cervical o absceso cervical asociado a compromiso obstructivo de vía aérea ${ }^{5,6}$. En la niñez y adolescencia se presenta como una masa cervical lateral; abscesos cervicales profundos, tiroiditis supurativa a repetición y absceso retrofarínge ${ }^{5-9}$. Los diagnósticos diferenciales a considerar son cualquier aumento de volumen cervical lateral tales como: seno de la $3^{\circ}$ bolsa faríngea, linfoadenitis, higroma quístico, tiroides ectópico, quiste tímico, linfoma, hemangioma, tumor del cuerpo carotídeo 3,5,6,8,9.

日 diagnóstico de esta anomalía requiere múltiples modalidades de estudio y un alto índice de sospecha. Ante la presencia de abscesos cervicales recurrentes y a tiroiditis supurativa recurrente que no responden a tratamiento convencional, que se presenta en la niñez y de preferencia en lado izquierdo debe plantearse esta anomalía como diagnóstico diferencial a tener en cuenta ${ }^{3-8}$.

日 inicio del estudio debe incluir un tránsito

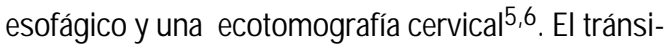
to esofágico tiene una sensibilidad de $80 \% 6$, este estudio evidenciará la presencia de un trayecto que se origina en el ápex del seno piriforme. Para que este examen tenga rendimiento es necesario que no exista infección recomendándose realizarlo después de 6 semanas del último episodio agudo. La ecotomografía cervical permite estudiar la presencia de una masa cervical pero falla en la visualización de senos y fístulas, siendo la presencia de gas un indicativo indirecto de apertura del seno piriforme. En caso de persistir con duda diagnóstica se recomienda realizar un TC cervical con y sin contraste y unaNR (evidenciar el orificio del seno de la IV bolsa faríngea). Si aún así no es posible plantear el diagnóstico con certeza y la clínica y los estudios son controversiales, es posible realizar una microlaringoscopía directa $3,5,6,8,9$.

日 tratamiento definitivo de un seno de la IV bolsa es esencialmente quirúrgico y consisteen identificar y disecar todo el trayecto del seno (para esto algunos autores recomiendan inyectar azul de metileno a la apertura del seno y otros cateterizan el trayecto con un catéter Fogarty), ligarlo y extraerlo3,5-9. Es importante retraer el lóbulo tiroideo hacia medial e incidir el músculo constrictor inferior para permitir una mejor visualización del trayecto así como la separación del cuerno inferior del cartílago tiroideo del cricoides para exponer el ápex del seno piriforme. Si el trayecto se encuentra en intima relación con el lóbulo tiroideo izquierdo se debe realizar una lobectomía ${ }^{5,6,8}$. La cirugía debe realizarse en un período libre de infección a menos que exista compromiso de vía aérea ${ }^{6,8}$. Las principales complicaciones quirúrgicas son la parálisis del nervio laríngeo recurrente y fístulas esófago-cutáneas ${ }^{5,9}$.

Otra alternativa terapéutica más reciente es la cauterización endoscópica, técnica que consiste en la obliteración del trayecto del seno utilizando electrocauterización. Este procedimiento es ambulatorio y posee menos riesgos que el procedimiento quirúrgico tradicional, disminuyendo al mínimo la posibilidad de lesionar estructuras tales como el nervio laríngeo superior, nervio laríngeo recurrente, esófago y tráquea. La indicación actual incluye a aquellos pacientes con trayecto corto y en aquellos casos en los que existe el antecedente de múltiples abscesos cervicales ${ }^{4,10}$.

\section{CONCLUSIÓN}

La presencia de IEPC recurrente en pacientes jóvenes, de preferencia en lado izquierdo y que no remite completamente con terapia clásica (antibioticoterapia y/o drenaje) debe alertar al clínico respecto a una probable anomalía de la IV bolsa faríngea $\mathrm{日}$ estudio inicial estará constituido por un tránsito esofágico y una ecotomografía cervical. La TC y NF son estudios en caso de duda diagnósti$\mathrm{ca}$

日 diagnóstico de certeza se realiza mediante una microlaringoscopía directa o una cervicotomía exploradora que permiten visualizar el orificio de salida del trayecto ciego en el seno piriforme y la relación de éste con el nervio laríngeo superior y recurrente.

日 tratamiento definitivo es esencialmente qui- 
rúrgico, el cual debe ser realizado en ausencia de infección aguda y consiste en identificar y resecar el trayecto del seno de la IV bolsa faríngea, en caso de compromiso de parénquima tiroideo se recomienda realizar una lobectomía para disminuir el riesgo de recurrencia.

\section{BIBLIOGRAFÍA}

1. Ungkanont $K$, Yellon R, Wessman J et al. Head and neck space infections in infants and children. Aolaryngol Head Neck Surg 1995; 112: 375-382.

2. NUSBAUM A, SOM P, ROTHSCHID M, SHUGAR J. Recurrence of a deep Neck Infection: A Cinical Indication $O$ an Underlying Congenital Lesion. Arch Oolaryngol Head Neck Surg 1999; 125: 1379-1382.

3. Godin M, Kearns D, Pransky S, Sed A, Wilson D. Fourth Branchial Pouch Sinus: Principles of Diagnosis and Management. Laryngoscope 1990; 100: 174-178.

4. Verret D, Mc Qlay J, Murray a, Biavati M, BRowN O. Endoscopic Cauterization of Fourth Branchial Cleft Sinus Tracts. Arch Otolaryngol Head Neck Surg 2004; 130: 465-468.

5. SHRIME M, KACKER A, BENT J, WARD R. Fourth branchial complex anomalies: a case series. Int
Una nueva alternativa terapéutica con resultados alentadores corresponde a la cauterización endoscópica, procedimiento ambulatorio y con menor morbilidad asociada que la cirugía tradicional.

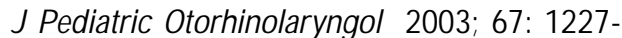
1233.

6. Liberman M, Kay S, EMIL S Et AL. Ten years of Experience With Third and Fourth Branchial Remnants. J Pediatr Surg 2002; 37: 685-690.

7. COTED, GANOLI G Fourth branchial cleft cysts. Otolaryngol Head Neck Surg 1996; 114: 95-97.

8. YANG C, COHeN J, EVertS E, SMITH J, CARO J, ANDERSEN P. Fourth Branchial Arch Sinus: Cinical Presentation, Diagnostic Workup, and Surgical Treatment. Laryngoscope 1999; 108: 442-446.

9. MANDEL D. Head and neck anomalies related to the Branchial apparatus. Oolaryngol Cin North Am 2000; 33: 1309-1332.

10. JORDAN J, GRAVES J, MANNING S, Mc QAY J, BiavaT M. Endoscopic Cauterization for Treatment of Fourth Branchial Cleft Sinuses. Arch Otolaryngol Head Neck Surg 1998; 124: 10211024.

Dirección: Dr. José Miguel Contreras.

Salvador 95 oficina 808, Santiago. Teléfono: 2690074.

Email: jmcontreras @alemana.cl 